\title{
Operacionalização do conceito de vulnerabilidade à tuberculose em alunos universitários
}

\author{
Functioning of the concept of vulnerability to tuberculosis \\ amongst university students
}

Alba Idaly Muñoz Sánchez ${ }^{1}$

Maria Rita Bertolozzi ${ }^{2}$

${ }^{1}$ Facultad de Enfermería, Universidad Nacional de Colombia. Carrera 45, 26-85, Edifício Uriel Gutierrez. Bogotá DC, Colombia. aimunoz@unal.edu.co ${ }^{2}$ Escola de Enfermagem, Universidade de São Paulo.
Abstract The objective of this study was to identify aspects that characterize the vulnerability to tuberculosis in resident students at the Residential Rooms of the University of Sao Paulo (CRUSP). The data relative to individual vulnerability were collected by using a questionnaire during the period of October to November, 2002. The theoretical reference and the interpretation frame to analize the issue of tuberculosis were given in the theorethical frame of vulnerability. The study sample completed 106 students being $69.8 \%$ graduated students and $30.2 \%$ postgraduated students. The proportion of emigrants (internal and external) reached $43.4 \%$. Seventy-nine students $(74.5 \%)$ answered that the disease can be cured. Nearly 60 (56.6\%) answered that they did not know the causal agent of the disease and 72 $(67.9 \%)$ said that they had already heard about tuberculosis, and for 5 of them (6.9\%) it happenned at health institutions. It was also asked if they knew where to ask in order to get tests to verify the presence of the disease and 48 (45.3\%) did not know. Although some results are positive it is identified the individual vulnerability of the students especially as they are apparently a well differentiated group, because in thesis they would have more favourable conditions to acced to the information related to the disease.

Key words Tuberculosis, Vulnerability, University students
Resumo O objetivo do presente estudo foi identificar aspectos que caracterizam a vulnerabilidade à tuberculose em moradores do Conjunto Residencial da Universidade de São Paulo (CRUSP). A população do estudo foi conformada por uma amostra estatisticamente significativa dos alunos universitários. Os dados relativos à vulnerabilidade individual foram coletados junto aos alunos no período de outubro a novembro de 2002. O referencial teórico para analisar a questão da tuberculose está pautado no quadro teórico de vulnerabilidade. A amostra totalizou 106 alunos, sendo 69,8\% de graduação e $30,2 \%$ de pós-graduação. A proporção de migrantes (internos e externos) alcançou 43,4\%. Sobre o conhecimento da tuberculose, 84 estudantes (79,2\%) responderam que sabiam o que era tuberculose e 79 (74,5\%) responderam que a doença tinha cura. Cerca de 60 (56,6\%) sabiam qual é o agente causal da doença e 72 (67,9\%) referiram já ter ouvido falar sobre a doença, sendo que para 5 (6,9\%) isso havia ocorrido em instituições de saúde. Indagou-se se sabiam onde recorrer para fazer exames para verificar a presença da enfermidade; 48 (45,3\%) responderam desconhecimento. Identifica-se a vulnerabilidade desses alunos, principalmente tendo em vista que constituem um grupo aparentemente diferenciado, pois em tese teriam condições mais favoráveis para acessar a informação a respeito da doença. Palavras-chave Tuberculose, Vulnerabilidade, Estudantes universitários 


\section{Introdução}

Os estudos em tuberculose mostram que o padrão de ocorrência da doença está relacionado fundamentalmente aos determinantes sociais, estruturados nos modos de produção e reprodução da sociedade. Nos últimos tempos, a situação da doença tem se agravado, em decorrência de certas políticas que vêm sendo adotadas, principalmente nos denominados países subdesenvolvidos, conforme se constata pelo incremento dos casos novos e número de mortes.

Diversos estudos têm identificado como mais vulneráveis para adquirir a doença as populações de baixa renda que, em geral, dispõem de condições de moradia insalubres, os portadores do HIV, os desnutridos, os imigrantes, além daqueles que têm acesso restrito aos bens básicos como saúde e educação ${ }^{1-5}$. Isto evidencia a associação entre as privações materiais e a presença da enfermidade, ademais dos indicadores clássicos, relacionados à doença e que, em geral, se apresentam como fatores, reduzidos a causas que promovem o seu aparecimento, através do aumento da exposição e da suscetibilidade à doença. Pretende-se, com o presente estudo, analisar a vulnerabilidade à tuberculose de um determinado grupo de pessoas, para disponibilizar informações que orientem os serviços de saúde na prestação da assistência.

Trata-se de um grupo que pode ser considerado como diferenciado, tendo em vista que se buscou estudar alunos de nível superior, residentes em um único local, o que possibilita condições privilegiadas de interpretação dos dados. O quadro conceitual da vulnerabilidade utilizado no presente estudo é o que vem sendo preconizado por Ayres et al. ${ }^{6}$. O estudo foi realizado por meio da aplicação de um instrumento contendo perguntas abertas e fechadas à parcela significativa do conjunto de pessoas selecionadas. Os resultados que se apresentam relacionam-se a algumas características pessoais dos sujeitos do estudo, assim como, especificamente, algumas questões relacionadas à vulnerabilidade individual, como o acesso aos processos comunicacionais e o conhecimento em relação à tuberculose.

O objetivo geral foi identificar aspectos que caracterizam a vulnerabilidade individual à tuberculose em moradores do Conjunto Residencial da Universidade de São Paulo (CRUSP).

\section{Metodologia}

\section{Marco referencial}

O referencial teórico para analisar a questão da tuberculose está pautado no quadro analítico da vulnerabilidade, que emerge no começo da década de 80 , para dar resposta à epidemia do $\mathrm{HIV}$, tentando recolocar a tendência individualizante, e que por isso imputava responsabilidade focalizada no individual, no controle da doença. O modelo de vulnerabilidade, proposto pioneiramente por Mann et al. ${ }^{7}$, apresenta uma metodologia para avaliar a vulnerabilidade à infeção pelo HIV e Aids. Segundo os autores, o comportamento individual é o determinante final da vulnerabilidade à infeção, o que justifica focalizar ações no indivíduo, embora isto não seja suficiente para o controle da epidemia. Deste modo, é importante considerar outros fatores que podem influenciar tal controle no âmbito individual. Os autores propõem uma estrutura de análise do conceito, em relação ao comportamento individual, que se refere à autoavaliação através de perguntas, para se verificar o conhecimento, os comportamentos, o status social e o acesso à informação, avaliando-se, em cada um desses eixos, como pode ocorrer a transmissão da doença; em relação à vulnerabilidade coletiva, que se refere à avaliação da capacidade estrutural e funcional dos programas de controle da epidemia; e em relação à vulnerabilidade social, que consiste na avaliação das realidades sociais através de indicadores do Programa de Desenvolvimento das Nações Unidas. Finalmente, os autores propõem a aplicação de um sistema de escores, que classificam a vulnerabilidade como alta, média e baixa. Nessa perspectiva, os autores enfatizam a necessidade de que os indivíduos se responsabilizem pela prevenção da doença, imputando a eles essa tarefa.

No Brasil, Ayres et al. ${ }^{8}$ apontam que o modelo de vulnerabilidade está conformado por três planos interdependentes de determinação e, consequentemente, de apreensão da maior ou da menor vulnerabilidade do indivíduo e da coletividade. O olhar dos autores busca a compreensão do comportamento pessoal ou a "vulnerabilidade individual", do contexto social ou "vulnerabilidade social" e do programa de combate à doença ou "vulnerabilidade programática". Cada um desses planos pode ser tomado como referência para se interpretar, também, outras enfermidades, além da Aids. Essa abordagem pode ampliar a atuação em saúde e gerar reflexões que 
podem ser úteis para continuar no caminho da formulação de políticas de saúde a partir das necessidades da coletividade, tendo em vista que propõe uma estrutura interpretativa que busca articular o indivíduo, num determinado contexto social, regido por certas políticas sociais e de saúde. Além disso, esse conceito coloca em tela a questão básica que se refere à cidadania como um exercício pleno de direitos.

O significado do termo vulnerabilidade, nesse caso, refere-se à chance de exposição das pessoas ao adoecimento como resultante de um conjunto de aspectos não apenas individuais, mas também que se referem ao coletivo, ao contexto, ao social, que acarretam maior suscetibilidade à infecção e ao adoecimento e, de modo inseparável, à maior ou menor disponibilidade de recursos de todas as ordens para a proteção das pessoas contra as enfermidades ${ }^{8}$. O marco conceitual que propõem os autores difere do marco inicial proposto por Mann et al. ${ }^{7}$, pois Ayres et al. ${ }^{8}$ não restringem a vulnerabilidade à determinação individual. Para esses autores, a unidade analítica está constituída no eixo indivíduo-coletivo.

Nessa perspectiva, Ayres et al. ${ }^{6}$ propõem a sua operacionalização através da "vulnerabilidade individual", que se refere ao grau e à qualidade da informação de que os indivíduos dispõem sobre os problemas de saúde, sua elaboração e aplicação na prática; da "vulnerabilidade social", que avalia a obtenção das informações, o acesso aos meios de comunicação, a disponibilidade de recursos cognitivos e materiais, o poder de participar nas decisões políticas e nas instituições; e da "vulnerabilidade programática", que se constitui na avaliação dos programas para responder ao controle de enfermidades, além do grau e qualidade de compromisso das instituições, dos recursos, da gerência e do monitoramento dos programas nos diferentes níveis de atenção.

Assim, este trabalho apresenta somente uma das faces do conceito de vulnerabilidade, pois se restringe aos aspectos que dizem respeito à vulnerabilidade individual. É necessário mencionar que este estudo integra um projeto-matriz que propõe o estudo da vulnerabilidade programática e da vulnerabilidade social. Desse modo, apresentam-se alguns dados relativos à vulnerabilidade individual à tuberculose, tendo em vista que essa enfermidade se constitui como uma das protagonistas no cenário epidemiológico brasileiro e, particularmente, do município de São Paulo. Assim, optou-se por estudá-la em um grupo restrito, que em tese teria acesso à informação, o que pode ser considerado no fortalecimento ao processo saúde-doença. Por esta razão, o estudo foi realizado com alunos moradores de um conjunto residencial de uma universidade.

\section{O cenário e os sujeitos de estudo}

O estudo foi realizado no Conjunto Residencial da Universidade de São Paulo (CRUSP) e se desenvolveu com alunos de graduação e de pósgraduação moradores deste local. A população foi conformada por uma amostra estatisticamente significativa, perfazendo um total de 106 estudantes. Os dados foram coletados através da aplicação de um formulário, que continha perguntas abertas e fechadas.

\section{Resultados}

\section{Características pessoais dos moradores do CRUSP}

\section{Idade e sexo}

A maior parcela de moradores pertence ao sexo masculino, com 62 pessoas $(58,5 \%)$. A faixa etária predominante das mulheres foi de 20 a 25 anos, conformada por 21 pessoas, representando $47,7 \%$ do total, e para os homens foi a imediatamente seguinte, de 25 a 30 anos, com 26 pessoas $(41,9 \%)$. Do conjunto total de alunos, verificou-se que a maior parte se concentrava nessa última faixa referida, o que configura uma população bastante jovem.

Os estudos em tuberculose mostram que a faixa etária de maior vulnerabilidade para desenvolver a enfermidade é de 20 a 40 anos, o que corresponde à faixa etária que alberga o continente populacional em idade economicamente ativa.

No Brasil, a faixa etária em que se evidencia a maior concentração de casos de tuberculose é de 20 a 49 anos, sendo que $92,5 \%$ da população do presente estudo encontram-se nesta faixa etária ${ }^{9}$.

\section{Escolaridade}

Setenta e quatro estudantes $(69,8 \%)$ encontravam-se matriculados no curso de graduação, e 42 alunos (30,2\%) estavam cursando o nível de pós-graduação. As áreas de estudo a que estes alunos estavam matriculados eram: Humanas, que concentrou o maior número de estudantes, com 50,4\% do total (53 pessoas), seguida da área de Exatas, com 34 alunos (31,6\%) e, por fim, da área de Biológicas, com 18 estudantes (16,9\%). 


\section{Naturalidade}

A maior parte dos alunos havia nascido no estado de São Paulo, com sessenta pessoas $(56,6 \%)$. Trinta e seis pessoas (34\%) eram oriundas do próprio município de São Paulo, o que perfaz $60 \%$ do montante do estado. A proporção de migrantes foi bastante alta, alcançando 43,4\%, tendo sido observados dois tipos de migração, interna e externa. Considerando-se a migração interna, identificou-se que os estudantes eram originários de diversas regiões do país, sendo principalmente da região Sul, com 11,2\%, seguidos dos estudantes procedentes das regiões Sudeste, Nordeste e Norte. A migração externa foi de 18,8\%, proveniente de diversos países da América Latina, principalmente Peru, Colômbia e Bolívia, e de dois países do continente africano. Desses estrangeiros, 15\% (três pessoas) estavam em São Paulo por um período menor que um ano; $45 \%$ (nove) entre um e três anos, $15 \%$ (três) entre três e cinco anos, $10 \%$ (dois) entre cinco e seis anos e $15 \%$ (três) há mais de 12 anos. Esse dado é importante, pois os alunos podem permanecer por períodos prolongados sem o contato mais próximo com suas famílias, o que pode constituir-se como um desgaste no processo saúde-doença.

Essa situação de diversidade mimetiza o cenário da cidade de São Paulo, que se configura como uma metrópole, caracterizada pela modernização de atividades e expansão da desigualdade e da pobreza, com intensa mobilidade de fatores de produção, o que estimula importantes contrastes nos espaços sociais ${ }^{10}$. É indubitável a conexão entre esse quadro e a vulnerabilidade, já que, conforme afirma Spossati ${ }^{11}$, a cidade se mostra como um local de contrastes, onde a vulnerabilidade social e a violência se associam à vulnerabilidade territorial. O fato de São Paulo ter se convertido numa metrópole global torna mais complexo o acesso universal e equitativo à saúde de sua população, assim como o atendimento às suas necessidades, já que o aumento das desigualdades espaciais e sociais impõe novos desafios na implementação de políticas de saúde que devem ser coerentes com os processos e as dinâmicas sociais, espaciais e políticas que a metrópole exige ${ }^{12}$. O processo migratório dos alunos não se aparta dos dados gerais do processo migratório nacional, e se apresenta diante de nós de forma clara, como uma característica importante a considerar neste grupo social. Segundo Santos, cada lugar revela um mundo, já que todos os lugares são suscetiveis de intercomunicação [...]. O lugar é o encontro entre as possibilidades latentes e as oportunidades preexistentes ${ }^{10}$.

\section{Acesso à informação}

Passar-se-á a analisar os dados relativos à questão da comunicação, categoria que fundamenta a vulnerabilidade individual e social, já que a partir do acesso aos meios de comunicação os indivíduos podem obter informação e, com isso, potencializar seu fortalecimento biopsíquico e adotar comportamentos e hábitos saudáveis no cotidiano.

É bem conhecido que a USP dispõe de uma série de equipamentos para a disseminação da comunicação voltados a sua comunidade interna, assim como à sociedade em geral, como por exemplo a Rádio USP, as atividades de extensão universitária, as apresentações da orquestra sinfônica, do coral, museus, entre outras. Assim mesmo, é importante analisar este aspecto porque ainda encontramos limitações em termos de acesso à informação, conforme verificar-se-á a seguir.

Encontrou-se que 77 (72\%) dos alunos tinham acesso à Internet com frequência de "sempre”, sendo esse meio de comunicação o mais usado pelos estudantes. Com menos expressão, e ainda na frequência de "sempre", 48 estudantes $(45,3 \%)$ referiram ouvir rádio, $43(40,6 \%)$ liam livros, 38 (35,8\%) assistiam televisão. As menores proporções foram encontradas no que se refere à leitura de jornal, com 34 (32,1\%) alunos, e referiram ler revistas não relacionadas à atividade escolar $23(21,7 \%)$. Na frequência de "algumas vezes”, o meio de comunicação mais acessado foi a televisão, com 64 estudantes $(60,4 \%)$, seguido pela leitura de jornal, por $61(51,7 \%)$ estudantes; de revistas, por 58 (54,7\%); de livros, por $46(43,6 \%)$; e acesso à Internet, por 27 $(25,5 \%)$. Um dado que chama a atenção nesta parcela populacional é que há estudantes que referiram nunca acessar esses meios de comunicação, sendo que 23 alunos $(21,7 \%)$ referiam que nunca liam revistas, 17 (16\%) nunca liam livros de literatura e o mesmo montante referiu não ouvir rádio; dez $(9,4 \%)$ nunca liam jornal, três $(2,8)$ nunca assistiam televisão e dois $(1,9 \%)$ mencionaram que nunca acessavam a Internet.

Alguns dos motivos expressos pelos alunos para a frequência de "às vezes" e "nunca" estavam relacionados ao fato de gostar ou não da atividade. Outro fator que influiu nas respostas de "algumas vezes”, em um meio tão massivo como a televisão, foi o fato de que alguns estudantes só têm acesso a esse meio de comunicação quando visitam seus familiares, e não é raro que isso ocorra com frequência muito reduzida para alguns estu- 
dantes. Também se evidenciou que as dificuldades econômicas afetam o acesso a esses meios de comunicação, já que alguns estudantes manifestaram obstáculos para a aquisição de livros necessários para seu próprio estudo. Vale dizer que alguns estudantes se deparavam com dificuldade para acessar a Internet, devido à limitação dos horários na sala de informática, nas unidades de ensino onde estudavam e no próprio CRUSP, uma vez que os apartamentos não têm conexão com a Internet. É importante destacar, ainda, que muitos estudantes referiram não possuir computador. Evidentemente que, para qualificar ainda mais a questão referente ao acesso aos meios de comunicação, seria necessário ter elencado outras perguntas para os alunos, relacionadas à propriedade de bens materiais que possibilitam esse acesso ao rádio e à televisão, entre outros meios de comunicação.

\section{Conhecimento da doença}

Em relação às questões relacionadas ao conhecimento dos alunos do CRUSP sobre a tuberculose, identificou-se que 84 pessoas $(79,2 \%)$ responderam que sabiam o que era a doença. Ao se solicitar para falar acerca do que sabiam da enfermidade, 81 pessoas $(76,4 \%)$ apontaram definições gerais sobre a doença; por exemplo: "doença do pulmão causada por um bacilo/bactéria”, “é uma doença transmissível”, “doença pulmonar infecciosa", "doença que ataca os pulmões causando deterioração dos mesmos", "doença que causa uma tosse insuportável”, "doença que começa com tosse que pode ocasionar a morte", "doença contagiosa que atinge os pulmões", "doença produzida pelo bacilo de Koch”, “doença que atinge o pulmão, se tratada precocemente tem cura" "doença difícil, que dana os pulmões".

Embora o número de pessoas que não sabiam o que era tuberculose tenha sido menor (22), é necessário destacar algumas das respostas: "doença que já não existe”, "doença com tosse que não sara, produzida por um vírus", "doença que atingiu muito a população no passado" ou "doença dos carentes".

Em relação à identificação da causa da enfermidade, a maioria dos estudantes participantes da pesquisa, $60(56,6 \%)$, responderam que não sabiam. Veja-se que algumas das pessoas que acreditavam conhecer a causa da enfermidade responderam da seguinte maneira: "doença produzida por vírus", "doença produzida por friagem” ou "complicação dos resfriados".
Ao se indagar se a tuberculose se transmitia de uma pessoa para outra, 81 pessoas $(76,5 \%)$ responderam afirmativamente, e quando questionados a respeito da transmissão, as respostas foram: através da via aérea, 55 (51,9\%); através do ar e de objetos pessoais, 16 (15,1\%), através de compartilhar objetos pessoais, $10(9,4 \%)$; não souberam responder, 17 (16\%), e 7,6\% referiram outras respostas.

Identificou-se, ainda, que 83 alunos (78,3\%) responderam que a doença tinha cura, $79(74,5 \%)$ responderam que a cura podia ser obtida através de medicamentos e dois $(1,9 \%)$ responderam que a cura poderia ser conseguida através de outras medidas, como a partir da vivencia em uma estação climática, e dois $(1,9 \%)$ não souberam responder.

No que se relaciona "a quem" os estudantes acreditavam que podiam contrair a doença, as respostas predominantes foram: "qualquer um", com 40,6\% de alunos (43). Onze alunos (10,3\%) responderam que "pessoas com baixas defesas" poderiam contrair a doença; 8,5\% (9) responderam que "pessoas pobres que vivem em condições insalubres", $7,5 \%$ (8) aqueles "expostos ao bacilo com pessoas infetadas", sete alunos $(6,6 \%)$ apontaram que os "boêmios", 11,3\% (12) não sabiam e outras respostas foram cerca de 15,2\%.

A respeito das pessoas que, segundo os estudantes, teriam mais chance de desenvolver a doença, se evidencia uma ampla opinião ao associar a doença com determinados estilos de vida (boêmios, fumantes), condição social (pobres), condição biológica (crianças, idosos) e condições de moradia e de trabalho insalubres, o que evidencia o estigma que a doença ainda carreia. Bertolozzi ${ }^{13}$ identificou um caráter moralizador nos doentes de seu estudo, em que o processo saúde doença assumiu um papel de ordenador da vida das pessoas, que passavam a deixar de fumar, de beber, o que pode ser uma resposta recorrente à forma de conceber a doença.

Assim, os conhecimentos insuficientes da doença podem afetar a forma de percebê-la e de enfrentá-la. É necessário resgatar os conhecimentos que a população têm sobre a saúde-doença para o planejamento de atividades que promovam a desmistificação de enfermidades como a tuberculose.

Uma questão interessante que surgiu neste estudo foi o fato de que, segundo o ponto de vista dos estudantes, "os boêmios" apresentam grande possibilidade de desenvolver tuberculose, e se observou ainda que os estudantes referiram a doença como própria de poetas e escritores, fato en- 
contrado por eles em alguns livros de literatura, o que pode ter influído nas associações no que se relaciona à transmissibilidade da doença e na definição de determinados grupos que supostamente teriam mais chance de contraí-la.

No presente trabalho, identifica-se como a tuberculose é assumida como uma "doença dos outros", do local e do trabalho insalubres, concepções tão antigas como a mesma doença.

Com relação ao tratamento, 14,2\% dos participantes não sabiam se a tuberculose tinha cura, o que reflete um desconhecimento importante, que ainda prevalece. Dessa forma, também podese interpretar esse resultado como a falta de informação acerca da tuberculose e a adoção de conceitos antigos. Outro fato relevante e que pode inferir nessas concepções é que somente 6,9\% dos moradores tinham ouvido sobre o tema em instituições de saúde. A maioria dos alunos tinha ouvido sobre o tema da tuberculose nas instituições de ensino, seguindo-se os meios de comunicação e a família/amigos.

Verifica-se a influência da sociedade na concepção da tuberculose, pelo pessoal de saúde e especificamente pelo setor educativo ${ }^{14}$, constatando-se que o âmbito educacional pode contribuir, por vezes, na mistificação da doença e no preconceito contra ela.

\section{Conclusões}

Identificaram-se algumas características pessoais e sociais como a faixa etária, a escolaridade, a migração, o acesso aos meios de informação e o conhecimento da doença em questão, que podem afetar a vulnerabilidade desse grupo populacional. Constata-se que os aspectos cognitivos acerca da doença parecem ser insuficientes. A tuberculose ainda é uma enfermidade caracterizada pelo preconceito, associada a modos de viver e trabalhar específicos. Assim, como pode se verificar neste grupo, que pode ser considerado como de incluídos, pois têm acesso ao espaço público da educação e da moradia, se evidenciam graus de exclusão ao acesso aos meios de comunicação, o que é paradoxal nesse local.
É necessário que se potencialize a acessibilidade aos meios de informação, uma vez que nos estudos sobre vulnerabilidade tem se evidenciado a importância do acesso aos processos comunicacionais na diminuição da suscetibilidade aos agravos e situações adversas.

Verifica-se que a concepção que os estudantes têm acerca da doença está influenciada pela forma como a sociedade historicamente a tem concebido, pois se observou uma "mistura" do higienismo (moradias e trabalho insalubres, falta de higiene), da concepção romântica (doença dos boêmios e escritores). Observaram-se, ainda, concepções que apontam a origem da enfermidade lastreada na Teoria da Unicausalidade (bacilo de Koch), da Multicausalidade (pobreza, alimentação inadequada, fumantes), sendo que alguns estudantes a associaram como doença determinada pela desigualdade da sociedade, o que indica a necessidade de promover informação para a diminuição do estigma da doença, o que pode ser obtido através dos aparelhos da sociedade, da saúde, da educação, da mídia etc.

A tuberculose continua a ser um sério problema de saúde e necessita de estratégias abrangentes para seu controle, o que faz necessário que os programas de controle da enfermidade revejam sua atuação, potencializando informação sobre a doença, através da televisão, do rádio e de outros meios de comunicação.

Cabe destacar o baixo percentual de pessoas que tinham ouvido falar sobre a tuberculose nas instituições de saúde, o que mostra, em parte, o reduzido impacto das atividades de promoção e prevenção da doença.

Ainda neste estudo se identifica a importância do trabalho intersetorial, já que a maioria dos alunos tinha ouvido falar sobre a doença através de instituições de ensino, o que demonstra como o processo de educação em saúde deve ser trabalhado pelos diferentes setores da sociedade, buscando desmistificá-la, evitando a perpetuação do seu estigma nas diferentes esferas da sociedade.

Finalmente, se identificaram questões que vão além da vulnerabilidade individual e transcendem a vulnerabilidade programática, dizendo respeito, sobretudo, à vulnerabilidade social. 


\section{Colaboradores}

EAIM Sánchez e MR Bertolozzi participaram igualmente de todas as etapas da elaboração do artigo.

\section{Referências}

1. Antunes JL, Waldman EA. The impact of Aids, immigration and housing overcrowding tuberculosis deaths in São Paulo, Brazil, 1994-1998 [serial online] 2001; 52:1071-1780. [acessado 2001 nov 11]. Disponível em: http//www.elsevier.com

2. Elender F, Bentham G, Langford I. Tuberculosis mortality in England and Wales during 1982-1992: its association with poverty, ethnicity and Aids. Soc Sci Med [periódico online] 1998; 46(6):673-681. [acessado 2001 jul 11]. Disponível em: http//www.elsevier. com

3. Khan A, Walley J, Newell J, Imdad N. Tuberculosis in Pakistan: socio-cultural contraints and opportunities in treatment. Soc Sci Med [periódico online] 2000; 50(2):247-254. [acessado 2001 nov 5]. Disponível em: http//www.elsevier.com

4. Macallan DC. Malnutrition in tuberculosis. Diagn Microbiol Infect Dis [periódico online] 1999; 34:153157. [acessado 2001 nov 3]. Disponível em: http// www.elsevier.com

5. Wallace DN. Discriminatory public policies and the New York City tuberculosis epidemia 1975-1993. Microbes Infection 2001; 3:515-524.

6. Ayres J, França I, Calazans G, Salletti H. Vulnerabilidade e prevenção em tempos de Aids. In: Barbosa R, Parker R, organizadores. Sexualidade pelo avesso: direitos, identidades e poder. Rio de Janeiro: Relume Dumará; 1999. cap. 34, p. 50-71.

7. Mann J, Tarantola DJM, Netter T. Como avaliar a vulnerabilidade à infeção pelo HIV e Aids. In: Parker R, organizador. A Aids no mundo. Rio de Janeiro: Relume Dumará; 1993. p. 276-300.

8. Ayres J, França I, Calazans G. Vulnerabilidade do adolescente ao HIV/Aids. In: Vieira EM, Fernandes MEL, bailey P, McKay A. Seminário gravidez na adolescência. Rio de Janeiro: Associação Saúde da Família; 1998. p. 97-109.

9. Melo FA. Etiologia e transmissão da tuberculose. In: Veronessi R, organizador. Tratado de infectologia. São Paulo: Atheneu; 1997. p. 915-916.

10. Santos M. Técnica espaço tempo: globalização e meio técnico-científico informacional. São Paulo: Hucitec; 1994.

11. Spossati A. Cidade em pedaços. São Paulo: Brasiliense; 2001.

12. Bousquat A, Nascimento VB. A metrópole paulista e a saúde. Rev São Paulo Perspec [periódico online] 2001; 15(1): [cerca de 9p.]. [acessado 2001 nov 5]. Disponível em: http://www.scielo.br/scielo.php? script $=$ sci_arttext\&pid=S0102-88392001000100013\& $\operatorname{lng}=$ pt\&nrm $=$ iso

13. Bertolozzi MR. Pacientes com tuberculose pulmonar no município de Taboão da Serra: perfil e representações sobre a assistência prestada nas Unidades Básicas de Saúde [dissertação]. São Paulo: Faculdade de Saúde Pública da USP; 1991.

14. Bertolozzi MR. A adesão ao programa de controle da tuberculose no Distrito Sanitário do Butantã, São Paulo [tese]. São Paulo: Faculdade de Saúde Pública da USP; 1998.

Artigo apresentado em 18/06/2007

Aprovado em 14/11/2007

Versão final apresentada em 14/12/2007 